\title{
Management im Umbruch
}

\author{
Armin Wöhrle
}

\begin{abstract}
Das Sozialsystem ist im Umbruch; die Politik sucht nach einer neuen Steuerung. Für die Organisationen der Sozialwirtschaft entstehen Unübersichtlichkeit und Unsicherheit. Ihr Management muss mit neuen Konzepten reagieren. Gebraucht werden allerdings keine Patentrezepte, sondern man sollte die Logik des Wandels verstehen lernen, um daraus Konsequenzen für die angemessene Suchbewegung entwerfen zu können.
\end{abstract}

Die Tätigkeitsbereiche der Sozialwirtschaft und ihre Finanzierung waren in Deutschland über viele Jahrzehnte geregelt. Insbesondere hinsichtlich der Wohlfahrtsverbände hatten wir es in Abstimmung mit den staatlichen Institutionen mit einem korporatistischen System zu tun. Die Absprachen im Sinne einer vertrauensvollen Zusammenarbeit auf der Grundlage des Subsidiaritätsprinzips waren stabil.

Nun gerät seit einiger Zeit der Kontext ins Wanken. Nachdem die politischen Systemgrenzen gefallen sind und ein globaler Markt politisch angestrebt wird, nachdem in der Europäische Gemeinschaft der freie Markt von Waren, Dienstleistungen und Arbeitskräften festgeschrieben wurde und mit dem europäischen Wettbewerbsrecht staatliche Subventionen (sogar das Gemeinnützigkeitsprinzip durch die deutsche Monopolkommission) infrage gestellt wird, kann das korporatistische System nicht einfach fortgeschrieben werden.

Die Europäische Union will in den globalen weltwirtschaftlichen Prozessen einen vorderen Platz behalten. Für die Mitgliedsländer, deren soziale Sicherungssysteme auf dem Einkommen durch bezahlte Arbeit und möglichst der Vollbeschäftigung ausgebaut sind und in denen gleichzeitig demografische Faktoren negativ wirken (wie in Deutschland), bedarf es einschneidender Korrekturen, wenn nicht gar eines Systemwechsels. Die Sicherungssysteme gegen die Risiken der Arbeitslosigkeit und Krankheit sowie für einen gesicherten Lebensabend funktionieren nicht mehr bei hoher Arbeitslosigkeit und langfristig ohnehin nicht, weil die Menschen länger leben, länger Rente beziehen, eine Krankenversorgung und Pflege benötigen, aber immer weniger junge Menschen geboren werden und die weniger werdenden Erwerbstätigen immer mehr Lasten der Sozialsysteme schultern müssen. Einer ständigen wachsenden Neuverschuldung, die Lasten auf künftige Generationen verschiebt, hat zudem die Europäische Gemeinschaft eine Grenze gesetzt.

In Europa wird auf die gleichen sozialen Probleme mit unterschiedlichen sozialpolitischen Systemen reagiert. Wir unterscheiden: Économie Sociale in Frankreich, Asso-

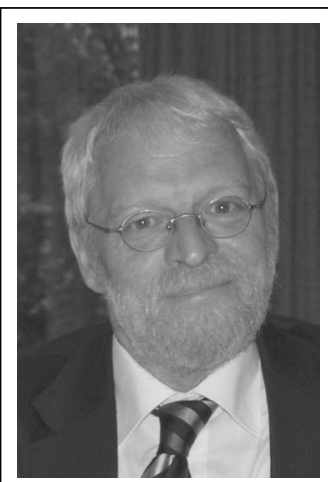

Prof. Dr. Armin Wöhrle ist Industriekaufmann, DiplomSozialpädagoge (FH) und Diplom-Pädagoge. Er hat langjährige Erfahrung als Organisationsberater, Fortbildner und Supervisor. Als Hochschullehrer für Sozialmanagement ist er auch im Leitungsbereich der Hochschule Mittweida tätig. Er ist Mitglied im erweiterten Vorstand der Bundesarbeitsgemeinschaft Sozialmanagement/Sozialwirtschaft.

E-Mailwoehrle@htwm.de

zianismus in Italien, Local Compact und New Deal in England, das ausgedehnt staatliche schwedische und das deutsche Modell (Besonderheit: das Subsidiaritätsprinzip). Die Modelle entspringen drei Quellen, denn Lebensrisiken können grundsätzlich abgesichert werden durch:

- das Individuum, das für sich selbst und seine Nachkommen vorsorgt (durch Privatversicherung, Sparen, Erbe etc.) - hierauf stützt sich das liberalistische Modell

- die Gesellschaft, in der durch Hilfe in der Nachbarschaft, im Sozialraum, durch Spenden, ehrenamtliche Tätigkeit, Stiftungen, Sponsoring etc. ein Ausgleich bei Hilfsbedürftigkeit geschaffen wird - hierauf stützen sich Modelle des bürgerschaftlichen Engagements, die im angelsächsischen Raum verbreitet sind

- den Staat, der einen Rechtsanspruch für die Bürger in Notlagen garantiert - hierauf stützen sich Modelle im skandinavischen Raum.

Das deutsche System hatte immer schon Elemente, die sich aus allen drei Quellen speisten. Der Schwerpunkt lag auf der staatlichen Sicherung. Der Umbau des deutschen Systems im letzten Jahrzehnt ist kein genereller Systemwechsel, jedoch bezieht er mehr Elemente mit ein, die einer anderen als der bisherigen Logik folgen. So wird beispielsweise gefördert, dass junge Berufstätige mehr individuelle Vorsorge treffen (»Riester-Rente«) oder es wird verlangt, dass Hilfeempfänger ihren Willen demonstrieren, wieder selbstständig ihren Lebensunterhalt bestreiten zu wollen (»Fördern und Fordern « bei »Hartz IV «). 


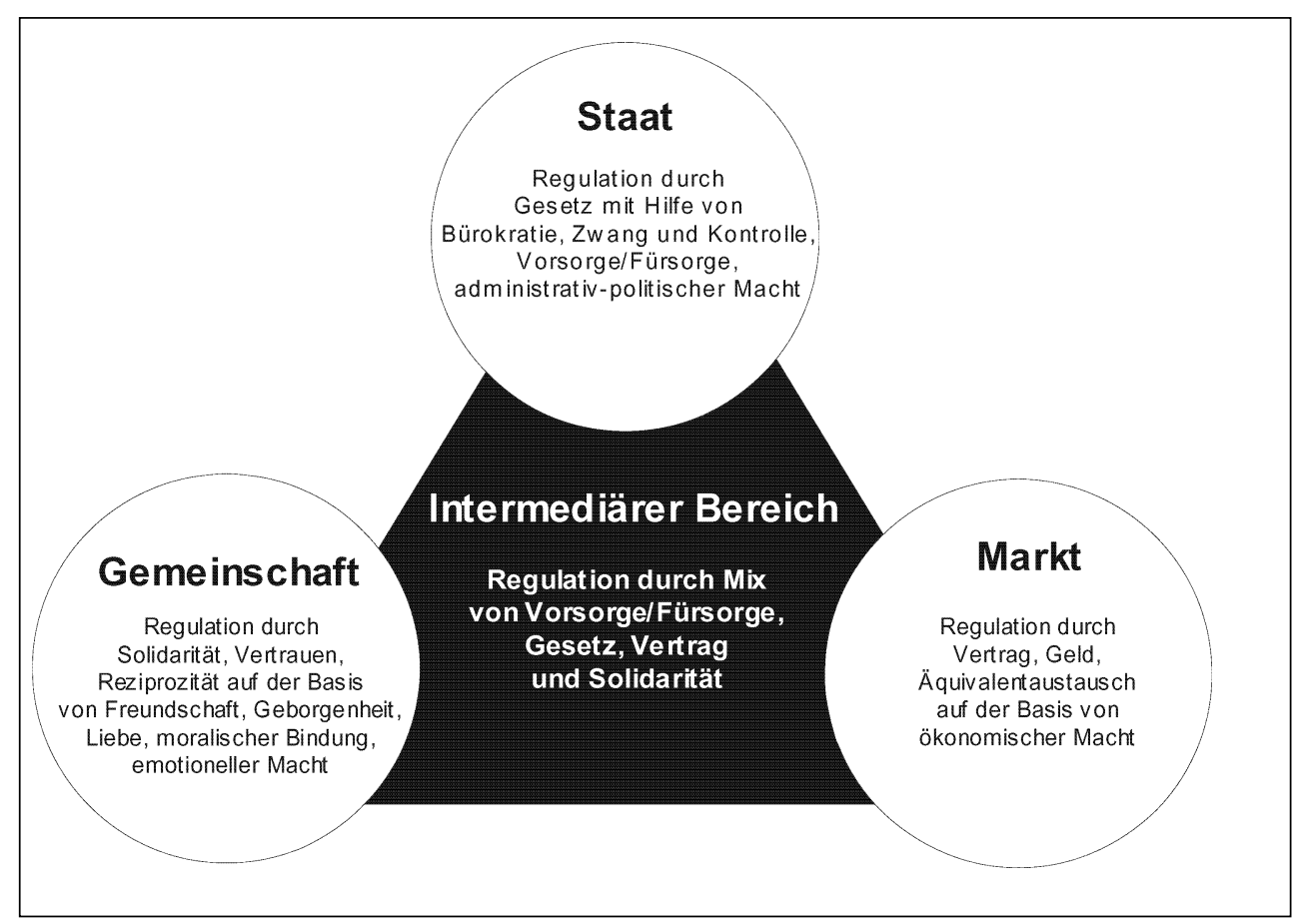

Abb. 1: Die soziale Sicherung kann sich aus verschiedenen Quellen speisen, von denen jede ihre eigene Logik hat.

(Darstellung analog der Grafik von: Effinger, H.: Sozialarbeitswissenschaft als Teildisziplin einer Wissenschaft personenbezogener Dienstleistungen im Wohlfahrtsdreieck, S. 191, in: Merten, R./Sommerfeld, P./Koditek, T. (Hg.): Sozialarbeitswissenschaft - Kontroversen und Perspektiven, Neuwied, Kriftel, Berlin 1996.) ge Arbeit als auch profitwirtschaftliche machen.

Eher künstlich wäre die Unterscheidung einer dritten Kategorie, die ausschließlich ehrenamtliche Leistungen erbringt und hierfür keine privaten Spenden und keine öffentlichen Mittel einwirbt.

Hinsichtlich sozialwirtschaftlicher Organisationen wird der Automatismus fest gefügter Angebots- und Abnahmestrukturen bei voller Übernahme der Kosten durch die öffentliche Verwaltung abgeschafft. Die alte Tradition der Verwaltung mit ihrer Bürokratie und Kameralistik wird auf ein neues Steuerungsmodell umzustellen gesucht. Es sollen marktwirtschaftliche Mechanismen und eine betriebswirtschaftliche Rechnung Einzug halten. Der Politik sollen mehr Mechanismen der Wirkungssteuerung zur Verfügung gestellt werden.

\section{Neue Steuerungslogik}

Das neue Sicherungssystem kann nach wie vor dreigeteilt gedacht werden. Wir haben es mit Steuerungsprozessen des Staates, der Gemeinschaft und der Wirtschaft im Bezugssystem der Organisationen zu tun, die Soziale Arbeit managen (vgl. Abb. 1). Gleichwohl gibt es hinsichtlich der Managementprozesse, die öffentliche und private Geldmittel bewirtschaften, lediglich zwei Akteure:

- Leitungsebenen in der öffentlichen Verwaltung, die politische Entscheidungen umzusetzen haben und

- das Management der Sozialwirtschaft, zu der die ganze Bandbreite der Non-Profit-Organisationen (die Vielfalt der kleinen gemeinnützigen Träger und die Wohlfahrtsverbände) zu rechen ist, aber auch die Betriebe, die profitwirtschaftlich arbeiten, sowie die steigende Zahl von Organisationen (für die noch keine eigenständige Bezeichnung existiert), die sowohl gemeinnützi-

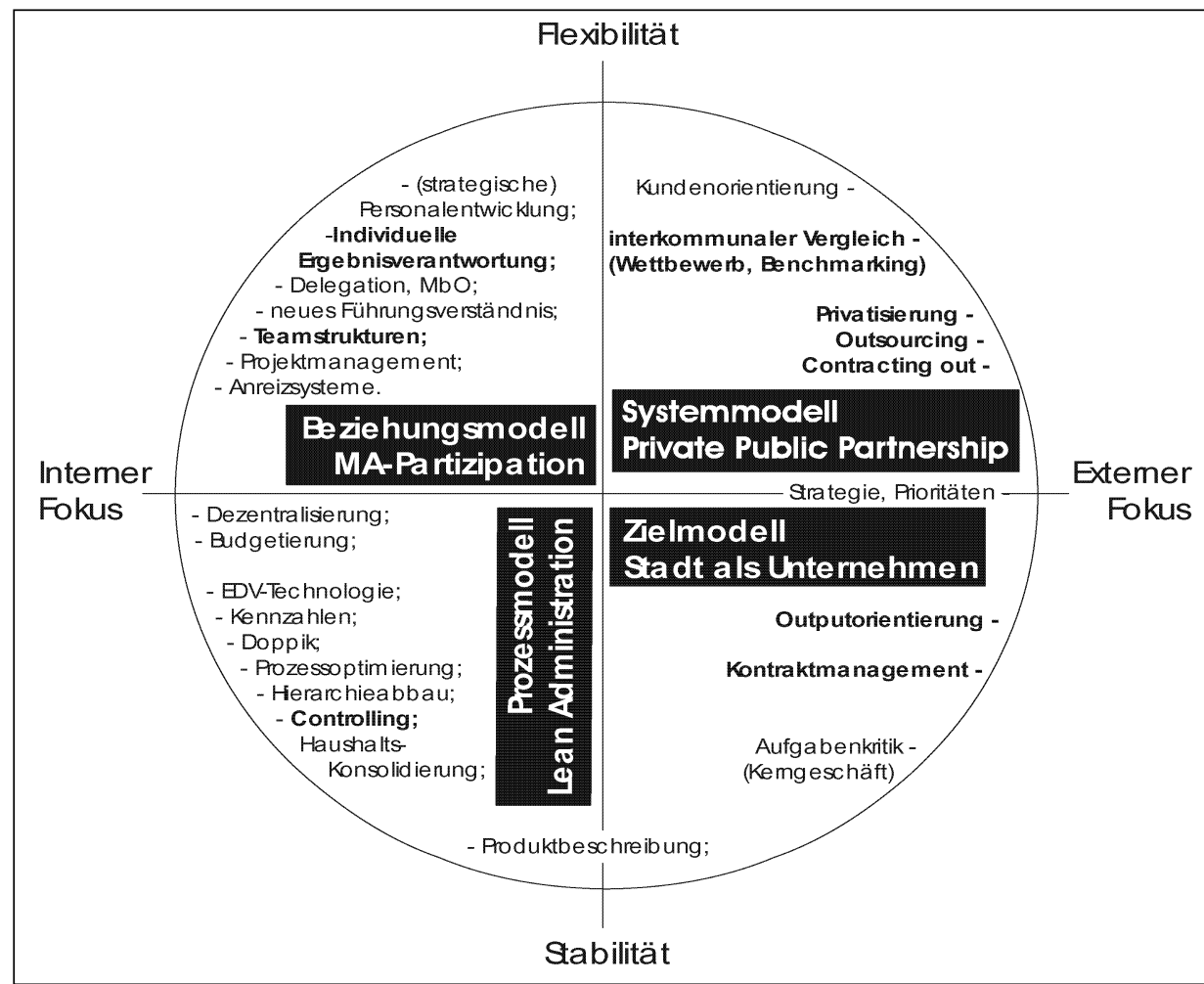

Abb. 2: Das Konzept der Neuen Steuerung der Kommunalen Gemeinschaftsstelle für Verwaltungsmanagement (KGSt) bildet eine der Grundlagen für die Modernisierung der Verwaltung und anderer großer Organisationseinheiten.

(Quelle: Morath, F. A./Altehage, M. A.: New Public Management. Ein neues Paradigma?, in: Morath, F. A.: Integrative Verwaltungsreform. Konzepte - Empirie - Erfahrungsberichte. Diskussionsbeitrag 1998 in der Reihe: Management, Forschung und Praxis (herausgegeben von R. G. Klimecki), Universität Konstanz 1998. Veröffentlicht im Internet http://www.ub.uni-konstanz.de/serials/klimecki.htm.) 
Im sozialwirtschaftlichen Leistungsdreieck wird damit die Nachfragermacht des Auftraggebers öffentliche Verwaltung gestärkt. Mit dem Instrument des Persönlichen Budgets soll zusätzlich die Nachfragermacht des Kunden, der die Leistung empfängt, gestärkt werden. Alle diese Umstellungen wirken deregulierend hinsichtlich des bislang fest gefügten korporatistischen Systems. Das Ziel ist, mehr Transparenz und mehr Verflüssigung in einem nahezu geschlossenen System zu erreichen, also mehr nachweisbare Effekte zu erzielen, womit die begrenzten Ressourcen zielgenauer eingesetzt werden können.

Das Management der Sozialwirtschaft muss im Interesse der Überlebenssicherung der Organisationen auf die Umstellung reagieren oder - noch besser - über die Wandlungsprozesse bei den Finanzgebern hinausdenkend, zu richtungsweisenden Managementmodellen kommen.

Die Generallogik für den Umstellungsprozess in der öffentlichen Verwaltung und im Management der Sozialwirtschaft kann gekennzeichnet werden als Abwendung vom bürokratischen und kameralistischen System und der Zuwendung zu betriebswirtschaftlichen Steuerungs- halb zu wendigen Fährbooten umgebaut werden müssen. Durchaus schwingt in diesem Bild mit, dass von vielen kleineren Einheiten auch ein paar in die Irre fahren können. Jedoch beschädigen sie damit das Ganze nicht nachhaltig. Dieses Modell bedeutet, dass bisherige Untergliederungen in den Verbänden in neue Rechtsformen überführt werden müssen, neue Managementstrukturen Einzug halten müssen, was zu Spannungen zwischen dem ehrenamtlichen und professionellen Potenzial führt.

3. Das dritte Bild ist die Antizipation der angemessen 3. Größe, um für die Märkte der Zukunft gewappnet zu sein. Im Blick auf große Träger geht es hier um Finanzvolumen, die aufgebracht werden müssen, um in einen Markt europäischen Zuschnitts einsteigen zu können. Bei kleinen und mittleren Trägern geht es darum, ab wann man sich Stellen leisten kann, die EU-Mittel einwerben. Kleine Träger mit einem ehrenamtlichen Vorstand und zwei Beschäftigten haben schon ein Problem, wenn jemand Urlaub nimmt oder krank wird. Hier kann keine betriebsinterne Fortbildung oder Supervision gesichert werden. Wenn man unter den neuen Bedingungen dabei sein will, muss zumindest mit anderen Kräften kooperiert werden, besser wäre jedoch über Fusionen nachzudenken, die eine angemessene Größe ermöglichen (vgl. Wöhrle, A.: Synergielösungen für Sozialräume. Plädoyer für Fusionen kleiner Träger. In: Blätter der Wohlfahrtspflege 4/2007). In großen Einheiten sollte man sich eine Forschungs- und Entwicklungsabteilung leisten können. mechanismen, wobei der für das Gesamtsystem sehr bedeutsame Steuerungsmechanismus des Rechts und dessen Besonderheiten hier ausgeklammert bleiben. Diese Generallogik des Wandels kann in Bildern angedeutet werden, die in den 1990er-Jahren Umstellungsprozesse der Wirtschaft in ihrer Reaktion auf die Globalisierung anleiteten und nun auch Richtschnur für die Sozialwirtschaft und öffentliche Verwaltung werden:

\section{1.}

Wesentlich für die öffentliche Verwaltung ist das 1. Bild der Verschlankung (Lean Management). Es beinhaltet den Abbau von »Wasserköpfen « (Entscheidungsebenen, die keine produktiven Einfluss auf Leistungen haben), die Entsäulung (Zusammenführung unterschiedlicher Entscheidungsebenen auf den Fall, der zu entscheiden ist) und die Aufhebung der Trennung von fachlicher und finanzieller Verantwortung. In diesem Bild können mittlere Entscheidungsebenen eliminiert werden. Es muss dezentralisiert werden, müssen Entscheidungsspielräume auf die unteren Ebenen verlagert und dort mehr Entscheidungsspielraum geschaffen werden. Es müssen interne Einheiten darauf überprüft werden, ob man sie für das Kerngeschäft wirklich braucht. Eine zentrale Beschaffungsstelle kann ersetzt werden, indem jede Abteilung selbst einkauft. Einrichtungen können ausgelagert werden, weil die Leistungen ein externer Anbieter erbringen kann und dabei kann Personal abgebaut werden. Das am deutlichsten ausgearbeitete Konzept hierfür ist das der Neuen Steuerung, das die Kommunale Gemeinschaftsstelle für Verwaltungsmanagement (KGSt) ausgearbeitet hat (vgl. Abb. 2).

2 Die Wohlfahrtsverbände und andere große Organi• sationen können als »Ozeanriesen « gesehen werden, die nicht auf jede Welle reagieren können und des-

\section{Ein neues Verständnis von Organisationen}

Neue Konzepte in der Organisationssoziologie kehren der Vorstellung den Rücken, Organisationen seien ausschließlich rationale Gebilde, die funktional und effektiv Ziele abarbeiten. Dieses neue Verständnis lenkt den Blick auf »Mikropolitik« und »Organisationskultur «. Damit entstehen neue Konzepte der »lernenden Organisation «, der »Organisationsgesellschaft « und damit einer neuen Kategorie des Managens, das die zentrale Ressource Personal stärker beachtet.

Für das Management interessant ist das Bild der »Delfinstrategien «, also des Sprungs aus dem Wasser, um einen Überblick im Meer des Wandels zu bekommen. Übertragen auf das Management in unübersichtlichem Gefilde heißt das: Die Führung muss sich vom Alltagsgeschäft entlasten und Frühwarnsysteme, Einflussnahmen in sozialpolitischen Zusammenhängen sowie intern eine neue Kultur und neue Steuerungsmechanismen ausbilden. Das Managementverständnis wird hierdurch einerseits anspruchsvoller, andererseits wird die Vorstellung hinsichtlich direkter Einflussnahme bescheidener. Die Kapazitäten des Personals werden deutlicher hervorgehoben, Wissensmanagement betrieben, sich selbst steuernde betriebliche Einheiten gewinnen an Bedeutung und das Management übernimmt eine mehr moderierende Funktion im internen Zusammenspiel bedeutsamer Kräfte.

Allen diesen Bildern und Modellen gemeinsam ist die Abkehr von geschlossenen Systemen, die Verflüssigung 
der Grenzen und der Ermächtigung des Personals zum Austausch mit der Systemumwelt, aber auch zur eigenständigen Entscheidung. Damit einher gehen steigende Anforderungen an die Qualifizierung des Personals. Sie muss auf allen Ebenen zunehmen und dies kann nicht ohne eine leistungsbezogene Vergütung erreicht werden.

\section{Resümee}

Die Situation eines umfassenden Umbruchs in allen wesentlichen Bezugssystemen und Veränderungen bedeutet für das Management jeder einzelnen Organisation zunächst Chaos. Die unmittelbare Reaktion ist, nach schnellen Lösungen zu suchen. Möglichst sollen Patentrezepte zur Verfügung stehen, die durch Berater mit wenigen Stunden Anleitung und in der Organisation mit wenigen Übersetzungsschritten eingebaut werden können. Eine Erkenntnis der Organisationssoziologie und Organisationspsychologie widerspricht diesem Impuls: Organisationen haben eher die Tendenz, sich zu geschlossenen Systemen auszubilden als zu wandeln.

Neuen Herausforderungen wird Widerstand entgegengebracht. Es wird nachzuweisen gesucht, dass die neuen

\section{Checkliste: Wie ist lhre Organisation für zukünftige Ent-} wicklungen gerüstet?

- Wann und unter welchen sozialpolitischen Rahmenbedingungen entstand Ihre Organisation? Welche Neuerungen hat sie mit welchen grundsätzlichen Umbrüchen verarbeitet? Hat sie Erfahrung mit grundsätzlichem Wandel?

- Wann wurde zum letzten Mal eine externe Instanz (Organisationsberatung, Untersuchung einer Hochschule etc.) hinzugezogen, um eine Organisationsanalyse durchzuführen?

- Hat Ihre Organisation eine Zukunftsvision, die über die nächsten Jahre hinausreicht?

- Wird das Leitbild, die Betriebsphilosophie von allen Organisationsmitgliedern getragen?

- Hat Ihre Organisation Personal für Forschung und Entwicklung oder kooperiert sie mit Instituten und Hochschulen?

- Welche Steuerungsinstrumente (z. B. Zielvereinbarungen, Qualitätsmanagement, Controlling, Wissensmanagement) setzen Sie ein und wie sind Sie damit zufrieden?

- Stehen neue Anforderungen an (z. B. neue Geschäftsbereiche, EU-Projekte, Umstellung der Förderrichtlinien), die neue Strukturen verlangen?

- Haben Sie belegbare Daten hinsichtlich der Kundenzufriedenheit, der Zufriedenheit der Finanzgeber und der Mitarbeiterzufriedenheit? Sind die Verfahren für entsprechende Rückmeldungen auf dem aktuellen Stand?

- Was haben Sie für ein Gefühl, nachdem Sie diese Fragen gelesen haben? Haben sie Sie eher verunsichert oder konnten Sie alles zu Ihrer Zufriedenheit abhaken?
Anforderungen nicht zur Organisation passen, was leicht nachzuweisen ist, weil die Organisation unter anderen Bedingungen entstanden ist und für die damaligen Bedingungen ihre Reaktionsfähigkeit ausgebildet hat. Anforderungen wird denn auch nicht selten nur auf einer oberflächlichen Ebene genüge getan. Ziele und Abläufe werden umetikettiert, mit modischen Namen versehen, aber man praktiziert das weiter, was immer getan wurde. Oder man veranstaltet ein großartiges Szenarium, das Bewegung suggeriert und veranstaltet am Ende einen hektischen Stillstand.

Organisationen können sich nur wandeln, wenn ihr Management für den Wandel offen ist. Nicht ausgeschlossen werden kann - und dies führt ja auch vorab schon zu Widerständen -, dass es in Wandlungsprozessen zu Krisen, Machtkämpfen, Verletzungen, Verschlechterungen für Organisationsmitglieder kommen kann. Dennoch darf dies nicht davon abhalten, die Organisation den neuen Herausforderungen auszusetzen: »Wir können Probleme nicht mit den Denkmustern lösen, die zu ihnen geführt haben.«(Albert Einstein)

Das Management sollte den Mut aufbringen, die Organisation der unabhängigen und wissenschaftlichen Betrachtung auszusetzen, aber sich auch der Wohltat angedeihen lassen, eine externe Beratung in Anspruch zu nehmen. Organisationen, die keine internen Forschungsund Entwicklungsabteilungen aufgebaut haben, können schwerlich diese Komplexität externen Anforderungen mit Mechanismen bisheriger interner Verarbeitung bewältigen.

\section{Literatur}

Wöhrle, A.: Change Management. Organisationen zwischen Hamsterlaufrad und Kulturwandel, Augsburg 2002.

Wöhrle, A.: Den Wandel managen. Organisationen analysieren und entwickeln, Baden-Baden 2005. 